OPEN ACCESS

Check for updates

\title{
The methodological quality of individual participant data meta-analysis on intervention effects: systematic review
}

\author{
Huan Wang, ${ }^{1}$ Yancong Chen, ${ }^{1}$ Yali Lin, ${ }^{1}$ Julius Abesig, ${ }^{1}$ Irene XY Wu, ${ }^{1}$ Wilson Tam ${ }^{2}$
}

${ }^{1}$ Xiangya School of Public Health, Central South University, 5/F, Xiangya School of Public Health, No. 238, Shang ma Yuan ling Alley, Kaifu district, Changsha,

Hunan, China

${ }^{2}$ Alice Lee Centre for Nursing Studies, National University of Singapore, Singapore

Correspondence to: IXYWU irenexywu@csu.edu.cn

(ORCID 0000-0003-3772-2376) Additional material is published online only. To view please visit the journal online.

Cite this as: $B M J$ 2021;372:n736 http://dx.doi.org/10.1136/bmj.n736

Accepted: 9 March 2021

\section{ABSTRACT}

OBJECTIVE

To assess the methodological quality of individual participant data (IPD) meta-analysis and to identify areas for improvement.

DESIGN

Systematic review.

DATA SOURCES

Medline, Embase, and Cochrane Database of Systematic Reviews.

ELIGIBILITY CRITERIA FOR SELECTING STUDIES

Systematic reviews with IPD meta-analyses of randomised controlled trials on intervention effects published in English.

\section{RESULTS}

323 IPD meta-analyses covering 21 clinical areas and published between 1991 and 2019 were included: 270 (84\%) were non-Cochrane reviews and 269 (84\%) were published in journals with a high impact factor (top quarter). The IPD meta-analyses showed low compliance in using a satisfactory technique to assess the risk of bias of the included randomised controlled trials (43\%, 95\% confidence interval $38 \%$ to $48 \%$ ), accounting for risk of bias when interpreting results ( $40 \%, 34 \%$ to $45 \%$ ), providing a list of excluded studies with justifications $(32 \%$, $27 \%$ to $37 \%$ ), establishing an a priori protocol (31\%, $26 \%$ to $36 \%$ ), prespecifying methods for assessing both the overall effects ( $44 \%, 39 \%$ to $50 \%$ ) and the participant-intervention interactions $(31 \%, 26 \%$ to $36 \%$ ), assessing and considering the potential of publication bias (31\%, $26 \%$ to $36 \%$ ), and conducting

\section{WHAT IS ALREADY KNOWN ON THIS TOPIC}

Individual participant data (IPD) meta-analysis is regarded as the ideal approach for providing evidence on intervention effect estimation but is susceptible to bias from methodological flaws

Evidence has shown that the published IPD meta-analyses were conducted based on inconsistent standards

Regardless of increasing numbers of published IPD meta-analyses, methodological quality has not been comprehensively evaluated

\section{WHAT THIS STUDY ADDS}

This study found that the methodological quality of IPD meta-analyses was unsatisfactory

IPD meta-analyses showed poor performance in general methodological items, especially in assessing and accounting for risk of bias of included trials, establishing an a priori protocol, assessing and considering the potential of publication bias, and conducting a comprehensive literature search

IPD meta-analyses showed poor performance in IPD specific methodological items, especially in prespecifying methods for data synthesis, checking data integrity, and undertaking strategy to account for unavailable IPD a comprehensive literature search $(19 \%, 15 \%$ to $23 \%)$. Up to 126 (39\%) IPD meta-analyses failed to obtain IPD from $90 \%$ or more of eligible participants or trials, among which only 60 (48\%) provided reasons and $21(17 \%)$ undertook certain strategies to account for the unavailable IPD.

\section{CONCLUSIONS}

The methodological quality of IPD meta-analyses is unsatisfactory. Future IPD meta-analyses need to establish an a priori protocol with prespecified data syntheses plan, comprehensively search the literature, critically appraise included randomised controlled trials with appropriate technique, account for risk of bias during data analyses and interpretation, and account for unavailable IPD.

\section{Introduction}

Well conducted systematic reviews with meta-analysis of randomised controlled trials are considered to be the best source of evidence on intervention effects. ${ }^{1}$ Meta-analysis is generally done by collecting aggregate data from publications or investigators. ${ }^{1}$ The aggregated data provide an average estimation of the intervention effect (eg, risk ratio or mean difference) in a group of patients with average characteristics (eg, diabetes diagnosis, mean age 50 years, 40\% women). ${ }^{2}$ This might limit the exploration of potential intervention-covariate interactions. Moreover, the validity of aggregate data meta-analysis is affected by the reporting quality of the randomised controlled trials and inconsistent definition of the outcomes across included trials. ${ }^{1}$ By collecting original data from the eligible primary studies, an individual participant data (IPD) meta-analysis has the ability to collect both published and unpublished data, derive standardised outcome definitions, use a consistent unit of analysis across included randomised controlled trials, and assess interactions between interventions and participants' characteristics. These advantages have led to the IPD meta-analysis being regarded as the ideal approach for providing evidence on intervention effect estimation. ${ }^{3}{ }^{4}$ IPD meta-analysis has shown substantial impact on clinical practice and research by informing the development of guidelines and design of randomised controlled trials. ${ }^{56}$ The number of yearly published IPD meta-analyses has increased over time, from eight in 1994 to 88 in 2014. ${ }^{4}$ These numbers, however, only refer to those IPD meta-analyses that were incorporated into systematic reviews-even larger numbers were published each year when IPD metaanalyses without systematic reviews were considered. ${ }^{4}$

The results from systematic reviews of metaanalysis, based on either aggregate data or IPD, are, however, not free from bias. ${ }^{7}$ Empirical evidence 
has indicated flaws when aggregate data metaanalysis are used for various medical conditions. ${ }^{9-12}$ Commonly reported problems include lack of a predefined protocol, comprehensive literature search, list of excluded studies with justifications, and check of funding information for the included studies. ${ }^{9-12}$ These in turn might threaten the validity of the evidence derived from systematic reviews. For instance, pre-established protocols and lists of excluded studies with justifications will prevent the exclusion of studies with unfavourable findings. ${ }^{17}$ It is, however, more difficult to design and conduct an IPD meta-analysis than aggregate data meta-analysis, and bias could affect the validity of the results. ${ }^{8}$ Published IPD meta-analyses have shown evidence of inconsistencies in methods used to estimate intervention effects (eg, the one stage method involving simultaneous analysis of IPD retrieved from eligible studies, and the two stage method, when IPD are first analysed separately for each study and then combined using a traditional meta-analysis method ${ }^{13}{ }^{14}$ ), how participant level covariates are assessed (eg, by participant subgroups, by trial subgroups, or using meta-regression), and whether trial variation was accounted for when combining IPD (eg, some IPD meta-analyses treated IPD from different trials as a mega-trial). ${ }^{15}{ }^{16}$ Such discrepancies suggest that evidence users such as researchers, clinicians, guideline developers, and policy makers should perform critical appraisal before applying evidence from IPD meta-analyses. Although IPD meta-analysis is a well established approach for synthesising evidence and having a direct impact on guideline development, methodological quality is still unclear.

In their paper, Tierney and colleagues offered guidance to evidence users on how to critically appraise the scientific rigour of IPD meta-analyses. ${ }^{2}$ They proposed eight key questions (composed of 31 signalling questions): four applied to IPD metaanalyses (questions 3, 4, 7, and 8) and the remainder to systematic reviews (questions 1, 2, 5, and 6). The eight key questions did not, however, cover some important methodological quality related components of systematic reviews, such as conflicts of interest and risk of bias of included studies. ${ }^{2}$ AMSTAR-2 (A MeaSurement Tool to Assess systematic Reviews-2) is a well developed, validated, and widely used tool for assessing the methodological quality of systematic reviews. ${ }^{7}$ It has been used critically appraise systematic reviews of various medical conditions. ${ }^{9}$ 17-19 AMSTAR-2 covers general methodological components of systematic reviews but has no specific item for assessing the unique methodological components of IPD meta-analysis. It has not been used to assess the methodological quality of IPD meta-analyses. We conducted a systematic review to describe the characteristics of an up-to-date sample of IPD meta-analyses, assess the methodological quality of the sampled IPD metaanalyses, and suggest areas for improvement in future IPD meta-analyses.

\section{Methods}

\section{Eligibility criteria}

An IPD meta-analysis was considered eligible for our study if it was included in a systematic review published before September 2019 and in English. We developed a practical criterion based on the definition of systematic review adopted in the Cochrane handbook. ${ }^{1} \mathrm{~A}$ systematic review had to provide eligibility criteria for study inclusion and conduct systematic literature searches in at least two databases. IPD meta-analyses of randomised controlled trials (considered the best source of evidence on intervention effect estimation) were considered eligible regardless of clinical areas studied. ${ }^{2021}$ To be considered as an IPD meta-analysis, data should have been obtained for quantitative synthesis either from the authors of randomised controlled trials or through other strategies such as data extraction from published trials.

We excluded IPD meta-analyses that summarised evidence from non-randomised controlled trials, quasi-randomised controlled trials, observational studies, diagnostic studies, prognostic studies, and economic evaluations, along with publications that focused on methodological issues with IPD, conference abstracts, and protocols. When an IPD meta-analysis was duplicated (ie, published in different journals) or one or more versions of the same IPD meta-analysis existed, we selected the most recent version; the others were used as supplementary documents for data extraction and critical appraisal.

\section{Literature search}

Using keywords related to IPD, we searched Medline, Embase, and the Cochrane Database of Systematic Reviews from inception to 30 August 2019. Specialised search filters for systematic reviews were adopted in Medline and Embase using the Ovid platform. ${ }^{22}{ }^{23}$ Our search strategies were based on a recent publication by Nevitt and colleagues, and we also extracted and screened citations included in that research. ${ }^{4}$ Appendix 1 provides details of our literature search strategies.

\section{Literature selection and data extraction}

Citations retrieved from both databases and Nevitt and colleagues' paper ${ }^{4}$ were screened and selected according to the eligibility criteria. We used a predeveloped and piloted data extraction form (see supplementary appendix 2) to retrieve data on basic characteristics (eg, year of publication) and other information such as IPD retrieval rate from each included IPD meta-analysis. Two researchers (HW, YL, JA, and YC) independently selected the literature and extracted data. Discrepancies were resolved by discussion and consensus or by referring to the original publications.

\section{Methodological quality assessment}

We are unaware of a specific tool for assessing the methodological quality of IPD meta-analyses on intervention effects. As such, we synthesised items from the two widely accepted criteria: AMSTAR-2 and 
Tierney and colleagues' guidance. ${ }^{27}$ When two criteria overlapped, we adopted the AMSTAR- 2 item if it captured the methodological components of IPD metaanalyses; otherwise we chose the item from Tierney and colleagues' guidance. All the non-overlapping items from AMSTAR-2 or Tierney and colleagues' guidance were included as they captured either the general methodological components of a systematic review or the specific methodological components of IPD meta-analysis. We also referred to other publications on methodological quality of IPD meta-analyses. ${ }^{24-26}$ A total of 22 items were included, with 15 adopted from AMSTAR-2, among these, six were considered as critical (table 1).

Supplementary appendix 3 provides detailed operational guidelines that were adopted from AMSTAR-2, Tierney and colleagues' guidance, or consensus among coauthors based on related publications. ${ }^{24-26}$ We tested the operational guidelines with a random sample of five IPD meta-analyses and revised accordingly. Two trained researchers (HW and YL) independently conducted the critical appraisal process. Disagreements were resolved by discussions and consensus. When agreement could not be reached, a senior researcher (IXYW) was consulted.

\section{Data analysis}

All collected data, including general information about the IPD meta-analyses and results of methodological quality assessments, are summarised descriptively. Basic characteristics of the IPD meta-analyses, detailed information, and critical appraisal results are presented as percentages with corresponding 95\% confidence intervals, or medians with interquartile ranges or ranges, as appropriate. Based on AMSTAR-2 and Jüni and colleagues' recommendations, we summarised the results for methodological quality assessments according to each item without generating an overall score. ${ }^{27}$ Compliance with each item is presented by year of publication to show the trends in methodological quality of the sampled IPD metaanalyses. IBM Statistical Package for Social Sciences (SPSS) 25 (IBM, Armonk, NY) was used for all data analyses.

\section{Patient and public involvement}

No patients were involved in conceiving the research question, choosing the outcome measures, or designing and implementing the study because of insufficient training, covid-19 related restrictions, and time constraints.

\section{Results}

A total of 15101 records were identified through database searches and reference lists (fig 1). Of these, 2197 remained after screening of the titles and abstracts, of which 1874 were excluded during full text assessment. The top three reasons for exclusion were not a systematic review ( $n=911)$, IPD meta-analyses included non-randomised controlled trials $(n=369)$, and a conference abstract $(n=295)$. Up to 323 (see supplementary appendix 4) IPD meta-analyses met the eligibility criteria and were included in this study.

\section{Basic characteristics of IPD meta-analyses}

The 323 IPD meta-analyses were published between 1991 and 2019 (median 2014; table 2). These covered 21 clinical areas according to ICD-11 (international classification of diseases, 11th revision) criteria (see supplementary appendix 5). The most studied conditions were neoplasia $(n=67,21 \%)$, diseases of the circulatory system $(n=64,20 \%)$, mental and behavioural or neurodevelopmental disorders $(n=31$, $10 \%)$, and diseases of the nervous system $(n=26$, $8 \%$ ). Most of the sampled IPD meta-analyses were non-Cochrane reviews $(\mathrm{n}=270,84 \%)$, published in the top quarter of high ranking impact factor journals $(n=269,84 \%)$, carried out by collaborative groups $(n=281,87 \%)$, and included a corresponding author from Europe $(n=246,76 \%)$. Among the 219 (68\%) IPD meta-analyses with funding support, 155 (71\%) were from Europe (table 2). The 323 IPD meta-analyses summarised evidence for drug interventions $(n=199$, $62 \%)$, non-drug interventions $(n=112,35 \%)$ (see supplementary appendix 6$)$, or both $(n=12,4 \%)$.

\section{Performing and reporting of IPD meta-analyses}

All IPD meta-analyses searched English databases, whereas only 17 (5\%) searched non-English databases. The most popular methods for pooling data were a two stage approach $(n=144,45 \%)$, both one and two stage approach $(n=96,30 \%)$, and one stage approach $(\mathrm{n}=75,24 \%)$. Three (1\%) IPD meta-analyses combined data as a "mega" trial (table 3). Supplementary appendix 7 provides details of the methods used for IPD meta-analyses. Only around half $(n=174,54 \%)$ of the IPD meta-analyses reported on harms related to interventions, with more IPD meta-analyses on drug interventions $(n=123,62 \%)$ reporting harms than IPD meta-analyses on non-drug interventions $(n=41$, $37 \%$ ) (table 2). Among the 310 IPD meta-analyses published in or after 2000 (a year after the publication of QUOROM (Quality Of Reporting Of Meta-analyses, the first reporting guideline for systematic reviews), only 91 (29\%) mentioned following any reporting guidelines (table 3 ).

\section{IPD retrieval rate}

A median of 11 (range 2-287) randomised controlled trials were included in systematic reviews, whereas the IPD were obtained from a median of seven (range $2-287$ ) trials, with a median proportion of $81 \%$ (range $8-100 \%)$ IPD obtained from included randomised controlled trials. A total of 90 (31\%) IPD meta-analyses obtained 100\% IPD from the included randomised controlled trials, 67 (23\%), 98 (34\%), and 37 (13\%) IPD meta-analyses obtained $80-99 \%$, 50-79\%, and less than 50\% IPD from the included randomised controlled trials, respectively. Among 230 IPD metaanalyses providing information on IPD retrieval based on participants level, 79 (34\%) obtained 100\% IPD from all eligible participants, 81 (35\%), 50 (22\%), and 


\section{Table 1 | Comparison of AMSTAR-2 and Tierney and colleagues' criteria for assessing the methodological quality of IPD meta-analyses and criteria used} in the current study

1. Did the research questions and inclusion

\section{AMSTAR-2 $^{7}$} criteria for the review include the components of PICO?

2. Did the report of the review contain an explicit statement that the review methods were established before conduct of the review, and did the report justify any significant deviations from the protocol?

3. Did the review authors explain their selection of the study designs for inclusion in the review?

4. Did the review authors use a comprehensive $1 \mathrm{~b}$. Does the IPD meta-analysis have a systematic literature search strategy?

\section{Tierney and colleagues ${ }^{2}$} question qualified by explicit eligibility criteria?

1c. Does it have a consistent approach to data collection? 1f. Has the protocol been registered or otherwise made available? 5. Were the analyses prespecified in detail? No related item and comprehensive search strategy? 1a. Does the IPD meta-analysis have a clear research 2a. Were fully published trials identified? $2 \mathrm{~b}$. Were trials published in the grey literature identified?

2c. Were unpublished trials identified?

5. Did the review authors perform study selection in duplicate?

6. Did the review authors perform data extraction in duplicate?
No related item

No related item 1e. Are all the methods prespecified in a protocol?

\section{Wang et al}

11. A clear research question is a general item for all systematic reviews

Item 1 from AMSTAR-2 was adopted

12. An a priori developed protocol is a general item for all systematic reviews. It is especially important for an IPD meta-analysis as it has the potential for a great number of analyses until desired results are obtained

Item 2 from AMSTAR-2 was adopted

13. Justification for inclusion of the study designs is a general item for all systematic reviews Item 3 from AMSTAR-2 was adopted

14. Comprehensive literature search is a general item for all systematic reviews

Item 4 from AMSTAR-2 was adopted

However, whether the IPD meta-analysis conducted searches within 24 months of completion of the review was excluded from the checklist as IPD meta-analyses generally take longer time than aggregate data meta-analyses

15. Duplicated study selection is a general item for all systematic reviews Item 5 from AMSTAR-2 was adopted

16. When basic characteristics and/or aggregate data are extracted from the included trials, duplicated data extraction will reduce manual mistakes and bias from subjective judgment Item 6 from AMSTAR-2 was adopted for all IPD meta-analyses unless it stated that no information was extracted from the publications 17. Providing a list of excluded studies with justifications will ensure transparency and it is a general item for all systematic reviews Item 7 from AMSTAR-2 was adopted

excluded studies and justify the exclusions?

No related item

8. Did the review authors describe the No related item

included studies in adequate detail?

18. Describing details about the PICO related information of included studies is a pre-requirement for judging whether the studies are appropriately selected for the research question. It is a general item for all systematic reviews Item 8 from AMSTAR-2 was adopted

9. Did the review authors use a satisfactory technique for assessing the RoB in individual studies that were included in the review?

10. Did the review authors report on the sources of funding for the studies included in the review?

11. If meta-analysis was performed, did the review authors use appropriate methods for statistical combination of results?

12. If meta-analysis was performed, did the review authors assess the potential impact of RoB in individual studies on the results of the meta-analysis or other evidence synthesis?

13. Did the review authors account for RoB in primary studies when interpreting/discussing the results of the review?

\section{Did the review authors provide a}

satisfactory explanation for, and discussion of any heterogeneity observed in the results of the review?

15. If they performed quantitative synthesis did the review authors carry out an adequate investigation of publication bias (small study bias) and discuss its likely impact on the results of the review?

16. Did the review authors report any potential sources of conflict of interest, including any funding they received for conducting the review? 1d. Does it assess the "quality" or RoB of included trials?

6a. Were randomisation, allocation concealment, and blinding assessed?

$6 \mathrm{~b}$. Were the IPD checked to ensure all (or most) randomised participants were included? $6 c$. Were all relevant outcomes included?

$6 \mathrm{~d}$. Was the quality of time-to-event-outcome data checked?

No related item

19. The RoB of included studies is the cornerstone for the quality of evidence generated from them. It is a general item for all systematic reviews. By combining the criteria from AMSTAR-2 and Tierney and colleagues, two questions were included

19-1. Item 9 from AMSTAR-2 was adopted. 19-2. $6 \mathrm{~d}$ from Tierney and colleagues was adopted

10. It is well established that certain funding source (eg, commercial funding) might introduce bias to the results. It is a general item for all systematic reviews Item 10 from AMSTAR-2 was adopted

7a-i. Did researchers stratify or account for clustering 111. An IPD meta-analysis requires certain special statistical techniques. of participants within trials using either a one or two stage approach to meta-analysis?

7a-ii. Was the choice of one or two stage analysis specified in advance and/or results for both approaches provided?

No related item Hence, Item 11 from AMSTAR-2 is not applicable Criteria $7 \mathrm{a}-\mathrm{i}$ and $7 \mathrm{a}$-ii from Tierney and colleagues were adopted as I11-1 and I11-2, respectively

112. Accounting for RoB of included studies during evidence synthesis is a general item for all systematic reviews Item 12 from AMSTAR-2 was adopted

No related item

113. Accounting for RoB of included studies when interpreting results is a general item for all systematic reviews Item 13 from AMSTAR-2 was adopted

7b-i. Did researchers compare treatment effects 114. Investigating possible source of trial level heterogeneity is between subgroups of trials or use meta-regression to important for identifying those patients who have the best chance to assess whether the overall treatment effect varied in benefit from the intervention. It is a general item for all systematic reviews relation to trial characteristics?

3b. Was an assessment of the potential impact of missing trials undertaken? Item 14 from AMSTAR-2 was adopted

115. Publication bias is a general item for all systematic reviews. Evidence suggested that many IPD meta-analyses neglected to examine or discuss publication bias Item 15 from AMSTAR-2 was adopted

No related item funding sources is a general item for all systematic reviews The item from AMSTAR-2 was adopted
I16. Potential sources of conflict of interest including 


\begin{tabular}{|c|c|c|}
\hline AMSTAR- $2^{7}$ & Tierney and colleagues ${ }^{2}$ & Wang et al \\
\hline No related item & $\begin{array}{l}\text { 3a. Were IPD obtained from a large proportion of the } \\
\text { eligible trials? } \\
\text { 3c. Were the reasons for not obtaining IPD provided? }\end{array}$ & $\begin{array}{l}\text { I17. This item is specific for IPD meta-analyses } \\
\text { Criteria } 3 \text { a and } 3 \text { c from Tierney and colleagues were adopted as I17-1 } \\
\text { and I17-2, respectively } \\
\text { Furthermore, suggestion on taking strategies to account for unavailable } \\
\text { IPD from Riley and colleagues }{ }^{24} \text { was adopted as } 117-3\end{array}$ \\
\hline No related item & $\begin{array}{l}\text { 4a. Were the data checked for missing, invalid, out of } \\
\text { range, or inconsistent items? } \\
4 \mathrm{~b} \text {. Were there any discrepancies with the trial report } \\
\text { (if available)? } \\
4 \mathrm{c} \text {. Were any issues queried and, if possible, } \\
\text { resolved? }\end{array}$ & $\begin{array}{l}\text { I18. These three items are specific to IPD meta-analyses } \\
\text { Criteria } 4 \mathrm{a}, 4 \mathrm{~b} \text {, and } 4 \mathrm{c} \text { from Tierney and colleagues were adopted as } \\
\text { I18-1, I18-2, and I18-3, respectively }\end{array}$ \\
\hline No related item & $\begin{array}{l}\text { 7c. Were the methods of assessing whether effects } \\
\text { of interventions vary by participant characteristics } \\
\text { appropriate? }\end{array}$ & $\begin{array}{l}\text { I19. This item is specific for IPD meta-analyses } \\
\text { Criterion } 7 \text { c from Tierney and colleagues was adopted as I19-1 } \\
\text { Furthermore, suggestions from PRISMA-IPD statement }{ }^{26} \text { and Fisher and } \\
\text { colleagues }^{25} \text { were adopted as I19-2 }\end{array}$ \\
\hline No related item & $\begin{array}{l}7 \mathrm{~d} \text {. If there was no evidence of a differential effect by } \\
\text { trial or participant characteristic, was emphasis placed } \\
\text { on the overall results? }\end{array}$ & $\begin{array}{l}\text { 120. This item is specific for IPD meta-analyses } \\
\text { The criterion from Tierney and colleagues was adopted. This item is } \\
\text { assessed when variation in treatment effect was explored in either trial } \\
\text { and participant characteristics level }\end{array}$ \\
\hline No related item & 7e. Were exploratory analyses highlighted as such? & $\begin{array}{l}\text { 121. This item is specific for IPD meta-analyses } \\
\text { The criterion from Tierney and colleagues was adopted }\end{array}$ \\
\hline No related item & $\begin{array}{l}\text { 8. Does any report of the results adhere to the PRIS- } \\
\text { MA-IPD statement? }\end{array}$ & $\begin{array}{l}\text { 122. This item is specific for IPD meta-analyses } \\
\text { The criterion from Tierney and colleagues was adopted and only applied } \\
\text { to those IPD meta-analyses that were published after } 2015 \text { (when the } \\
\text { PRISMA-IPD statement was published) }\end{array}$ \\
\hline
\end{tabular}

20 (9\%) IPD meta-analyses obtained 80-99\%, 50-79\%, and less than 50\% IPD from all eligible participants, respectively (table 3 ).

\section{Methodological quality}

The methodological quality of the 323 sampled IPD meta-analyses was generally unsatisfactory either on general items for systematic reviews (table 4) or on items specific to IPD meta-analyses (table 5). However, improvements were seen over time in most of the methodological items, especially in pre-establishing protocol and data analysis plan, accounting for risk of bias and publication bias (fig 2 and supplementary appendix 8).

\section{Critical appraisal results on general items}

Table 4 provides results for the critical appraisal on general items. The sampled IPD meta-analyses showed more than $80 \%$ compliance in three items-stating conflict of interests $(92 \%, 95 \%$ confidence interval $89 \%$ to $95 \%$ ), including PICO (population, intervention, comparator, and outcome) components in the research question and inclusion criteria (85\%, $81 \%$ to $89 \%$ ), and explaining the observed heterogeneity $(81 \%, 77 \%$ to $85 \%$ ). None of the items are, however, critical ones in AMSTAR-2.

The sampled IPD meta-analyses showed unsatisfactory performance for the six critical items in AMSTAR-2 that were applicable to IPD meta-analyses. Only $43 \%$ (38\% to $48 \%$ ) of IPD meta-analyses used a satisfactory technique for assessing the risk of bias of included randomised controlled trials (table 4). Ninety seven IPD meta-analyses (30\%, 25\% to $35 \%$ ) did not perform any critical appraisal of the included randomised controlled trials, and 56 (17\%,
$13 \%$ to $22 \%$ ) did not report the tool they used for critical appraisal (table 3). The sampled IPD metaanalyses showed no more than $40 \%$ compliance for the remaining five critical items-accounting for risk of bias when interpreting results (40\%, 34\% to $45 \%$ ), providing a list of excluded studies with justifications $(32 \%, 27 \%$ to $37 \%)$, establishing an a priori protocol and justifying any deviations (31\%, 26\% to $36 \%$ ), assessing and considering the potential of publication bias (31\%, 26\% to $36 \%$ ), and conducting a comprehensive literature search $(19 \%, 15 \%$ to $23 \%$; table 4).

The remaining five non-critical items in AMSTAR-2 that were applicable to IPD meta-analyses showed less than $50 \%$ compliance for the sampled IPD meta-analyses. Two items had no more than $20 \%$ of sampled IPD meta-analyses rated as yes: explaining the selection of the study design $(10 \%, 7 \%$ to $14 \%)$ and reporting sources of funding for the included randomised controlled trials $(18 \%, 14 \%$ to $22 \%)$.

\section{Critical appraisal results on items specific to IPD meta-analyses}

Except for stratifying or accounting for clustering of participants within trials (98\%, 96\% to 99\%) and using appropriated methods to assess whether effects of interventions varied by participant characteristics (71\%, 66\% to $76 \%$ ), the performance of the sampled IPD meta-analyses on specific items were generally unsatisfactory, with less than $60 \%$ showing compliance with each item. A relatively low proportion of the sampled IPD meta-analyses prespecified methods either for assessing the overall effects (44\%, 39\% to $50 \%$ ) or for assessing participant-intervention interactions $(31 \%, 26 \%$ to $36 \%)$. Furthermore, only 


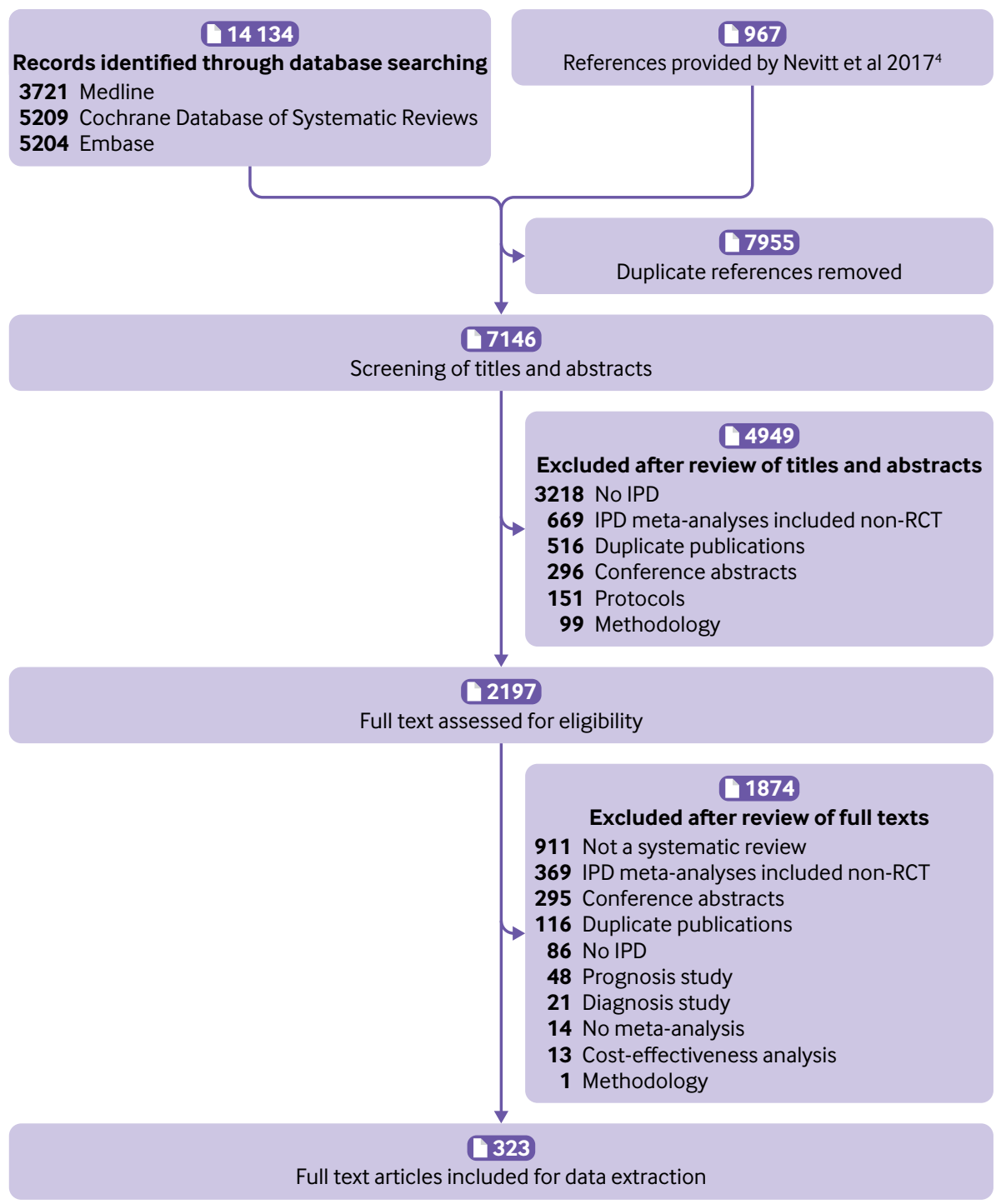

Fig 1 | Screening and selection process of individual participant data (IPD) meta-analyses. RCT=randomised controlled trials

$48 \%$ (42\% to $53 \%$ ) of the sampled IPD meta-analyses labelled the analyses as prespecified or post hoc, and $5 \%$ (3\% to $7 \%$ ) of exploratory analyses were not labelled as such. The remaining $47 \%$ (42\% to $53 \%$ ) did not provide related information (table 5).

Up to 126 (39\%, 34\% to 44\%) IPD meta-analyses failed to obtain IPD from $90 \%$ or more of eligible participants or trials, or both. Among them, only 60 (48\%, 39\% to 56\%) provided reasons for not obtaining IPD, and $21(17 \%, 10 \%$ to $23 \%)$ undertook certain strategies to account for the unavailable IPD. Only $56 \%$ (50\% to $61 \%$ ) of IPD meta-analyses checked for missing, invalid, out of range, or inconsistent items, and only $55 \%$ (50\% to $61 \%$ ) contacted trial authors for clarifications. Among the 127 IPD meta-analyses published after 2015, only 41 (32\%, 24\% to 40\%) reported that the PRISMA-IPD (Preferred Reporting Items for Systematic Reviews and Meta-Analyses for Individual Participants Data extension) was followed (table 5).

\section{Discussion}

This study identified and appraised 323 IPD metaanalyses of randomised controlled trials that focused on intervention effects. This sample of IPD meta-analyses (to August 2019) covered 21 different clinical areas and comprised Cochrane and non-Cochrane reviews as well as drug and non-drug interventions. According to our criteria, the methodological quality of the sampled IPD meta-analyses was far from satisfactory. In future IPD meta-analyses much consideration is needed of both general methodological components of systematic reviews (eg, establishing an a priori protocol, using a comprehensive literature search strategy, assessing the risk of bias of included randomised controlled trials with a satisfactory approach as well as accounting for risk of bias when interpreting results, and addressing potential publication bias) and IPD meta-analyses specific components (eg, prespecifying methods used for data analyses and putting a label for exploratory analyses, providing reasons for not obtaining IPD 


\begin{tabular}{|c|c|c|}
\hline Characteristics & No of IPD meta-analyses & $\%(95 \% \mathrm{Cl})$ \\
\hline Cochrane review & 53 & $16(12$ to 20$)$ \\
\hline Update of a previous review & 80 & $25(20$ to 30$)$ \\
\hline Median (range) publication year & 2014 & 1991-2019 \\
\hline Median (interquartile range) publication journal impact factor & 6 & $4-13+$ \\
\hline 1st (top) & 269 & $84(80$ to 88$) \ddagger$ \\
\hline 2nd & 34 & 11 (7 to 14$) \neq$ \\
\hline $3 r d$ & 11 & $3(1$ to 5$) \ddagger$ \\
\hline 4th (bottom) & 6 & $2(0.4$ to 3$) \ddagger$ \\
\hline \multicolumn{3}{|l|}{ Location of corresponding author: } \\
\hline \multicolumn{3}{|l|}{ Authorship: } \\
\hline Collaborative group & 281 & 87 (83 to 91) \\
\hline Individual authorship & 42 & $13(9$ to 17$)$ \\
\hline \multicolumn{3}{|l|}{ Type of funding: } \\
\hline Non-commercial & 161 & $50(44$ to 55$)$ \\
\hline Commercial & 38 & $12(8$ to 15$)$ \\
\hline Mixed & 20 & $6(4$ to 9$)$ \\
\hline No funding & 42 & $13(9$ to 17$)$ \\
\hline Not reported & 62 & 19 (15 to 24$)$ \\
\hline Funding location: & 219 & $68(63$ to 73$)$ \\
\hline Diseases of the circulatory system & 64 & $20(15$ to 24$)$ \\
\hline Mental, behavioural, or neurodevelopmental disorders & 31 & $10(6$ to 13$)$ \\
\hline Diseases of the nervous system & 26 & $8(5$ to 11$)$ \\
\hline \multicolumn{3}{|l|}{ Type of intervention: } \\
\hline Drug & 199 & $62(56$ to 67$)$ \\
\hline Non-drug & 112 & $35(30$ to 40$)$ \\
\hline Drug and non-drug & 12 & $4(2$ to 6$)$ \\
\hline IPD meta-analyses reported intervention harms: & 174 & $54(48$ to 59$)$ \\
\hline Drug intervention & 123 & $62(55$ to 69$) 9$ \\
\hline Non-drug intervention & 41 & 37 (28 to 46) \\
\hline Drug and non-drug & 10 & $83(62$ to 100$) \emptyset$ \\
\hline \multicolumn{3}{|c|}{$\begin{array}{l}\text { *Data are number of IPD meta-analyses, percentage ( } 95 \% \text { confidence interval) unless stated otherwise. } \\
\text { TThree IPD meta-analyses did not publish in a journal with impact factor. } \\
\text { ‡Denominator is } 320 \text {. } \\
\text { §Denominator is } 219 \text {. } \\
\text { १Percentages were calculated using total number of categories as denominator. }\end{array}$} \\
\hline
\end{tabular}

and taking strategies to account for unavailable IPD, checking data integrity, and clarifying uncertainties in need). Future IPD meta-analyses should also justify the study design for inclusion and report funding information for the included randomised controlled trials, as less than $20 \%$ of the sampled IPD metaanalyses complied with these two items.

\section{A priori protocol and exploratory analyses}

A predeveloped protocol will help increase objectivity and reduce bias in systematic reviews. ${ }^{728} \mathrm{~A}$ prespecified protocol with a detailed plan for data analyses is of importance to an IPD meta-analysis, because raw data collected from randomised controlled trials enables reviewers to perform many analyses and this poses the risk of data being repeatedly interrogated until desired results are obtained. ${ }^{1}$ However, only $31 \%$ of the sampled IPD meta-analyses established an a priori protocol and justified important deviations from the protocol. Conducting exploratory analyses to identify potential effect modifiers, at either the trial or the participant level is, however, a recognised advantage of IPD meta-analyses. ${ }^{1}$ Exploratory analysis therefore is not prohibited in IPD meta-analyses, and it enables the collection of extra information on certain subgroups of participants, who might benefit more from the intervention and contributes to better clinical decision making. Nonetheless, appropriate interpretation of the results from IPD meta-analyses requires full presentation of all the analyses, with exploratory analyses being labelled as such. It has been suggested that future IPD meta-analyses should predevelop 


\begin{tabular}{|c|c|c|}
\hline Characteristics & No of IPD meta-analyses & $\%(95 \% \mathrm{Cl})$ \\
\hline \multicolumn{3}{|l|}{ Language of databases searched: } \\
\hline English & 323 & 100 \\
\hline Non-English & 17 & 5 (3 to 8) \\
\hline \multicolumn{3}{|l|}{ Proportion of IPD retrieved from eligible RCTs (\%): } \\
\hline 100 & 90 & $31(26$ to 36$) \dagger$ \\
\hline $80-99$ & 67 & $23(18$ to 28$) \dagger$ \\
\hline $50-79$ & 98 & $34(28$ to 39$) \dagger$ \\
\hline$<50$ & 37 & $13(9$ to 16$) \dagger$ \\
\hline \multicolumn{3}{|l|}{ Proportion of IPD retrieved from eligible participants (\%): } \\
\hline 100 & 79 & $34(28$ to 40$) \neq$ \\
\hline $80-99$ & 81 & 35 (29 to 41$) \ddagger$ \\
\hline $50-79$ & 50 & 22 (16 to 27$) \ddagger$ \\
\hline$<50$ & 20 & $9(5$ to 12$) \ddagger$ \\
\hline Median No (range) of RCTs included in IPD meta-analyses & 7 & $2-287 \S$ \\
\hline Median No (range) of RCTs included in systematic reviews & 11 & $2-287$ \\
\hline Median No (range) of participants included in IPD meta-analyses & 1940 & 49-2120001 \\
\hline Median No (range) of participants included in systematic reviews & 2422 & $49-212000^{\star \star}$ \\
\hline Median proportion (range) of IPD retrieved from eligible participants (\%) & 93 & $8-100+t$ \\
\hline Median proportion (range) of IPD retrieved from RCTs (\%) & 81 & $8-100 \S$ \\
\hline \multicolumn{3}{|l|}{ Eligibility criteria based on language of publication: } \\
\hline Language criteria not reported & 138 & $43(37$ to 48$)$ \\
\hline English and non-English & 126 & $39(34$ to 44$)$ \\
\hline English publications only & 59 & 18 (14 to 22$)$ \\
\hline \multicolumn{3}{|l|}{ Tools used for RoB assessment of included RCTs: } \\
\hline Cochrane RoB tool & 145 & $45(39$ to 50$)$ \\
\hline Jadad scale & 12 & $4(2$ to 6$)$ \\
\hline Otherł‡ & 13 & $4(2$ to 6$)$ \\
\hline Not assessed & 97 & $30(25$ to 35$)$ \\
\hline Not clear which tool was used & 56 & $17(13$ to 22$)$ \\
\hline Followed any reporting guidelines for systematic reviews or IPD meta-analyses: & 91 & 29 (24 to 34$) \S \S$ \\
\hline QUOROM (1999) & 4 & $4(0.1$ to 9$)$ १ी \\
\hline PRISMA (2009) & 46 & 50 (40 to 61) १1 \\
\hline PRISMA-IPD (2015) & 36 & $40(29$ to 50$)$ १ฯ \\
\hline Both PRISMA (2009) and PRISMA-IPD (2015) & 5 & 6 (1 to 10)ाभा \\
\hline Methods used to combine IPD: & 318 & $98(97 \text { to } 100)^{\star \star \star}$ \\
\hline Two stage approach & 144 & 45 (40 to 51$)+t+$ \\
\hline One stage approach & 75 & 24 (19 to 28$)+t \dagger$ \\
\hline One and two stage approach & 96 & 30 (25 to 35$)+t t$ \\
\hline A "mega" trial & 3 & 1 (0 to 2$)+t \dagger$ \\
\hline
\end{tabular}

RCT=randomised controlled trial; QUOROM=Quality Of Reporting Of Meta-analyses; PRISMA=Preferred Reporting Items

for Systematic Reviews and Meta-analysis; PRISMA-IPD, Preferred Reporting Items for Systematic Review and Meta-

Analyses of individual participant data; RoB=risk of bias.

*Values are numbers of IPD meta-analyses, percentage ( $95 \%$ confidence interval) unless stated otherwise

tDenominator is 292 .

\#Denominator is 230

$\S 31$ did not report number of randomised controlled trials included in systematic reviews.

ๆ1 did not report number of participants included in IPD meta-analysis.

**92 did not report number of participants included in systematic reviews.

t+93 did not report number of eligible participants.

\# Included tool for the assessment of study quality and reporting in exercise $(n=3,1 \%)$, Delphi list $(n=2,1 \%)$, Chalmer scale $(n=2,1 \%)$, Effective Public Health Practice Project Quality Assessment Tool $(n=1,0.3 \%)$, Jüni $(n=1,0.3 \%)$, Pedro scale $(n=1,0.3 \%)$, Method for Evaluating Research and Guideline Evidence (MERGE) criteria $(n=1,0.3 \%)$, Consolidated Standards of Reporting Trials (CONSORT) statement $(n=1,0.3 \%$ ), and Cochrane RoB plus Pedro scale $(n=1,0.3 \%)$.

$\S \S$ Denominator is 310 IPD meta-analyses published in or after 2000.

११९1 IPD meta-analyses followed one or more reporting guidelines.

$\star \star \star 5$ IPD meta-analyses did not report the methods used to combine IPD.

††+318 IPD meta-analyses reported the methods used to combine IPD.

the research protocol, register it in PROSPERO or the Cochrane Library, and label exploratory analyses as such. 2262930

\section{Literature searches and publication bias}

The importance of a comprehensive literature search is well established in systematic reviews. ${ }^{1}$ Theoretically, IPD meta-analyses have advantages of comprehensively identifying literature, especially unpublished randomised controlled trials through collaboration with multiple research groups and consultation with trialists. ${ }^{1}$ However, we found that only $19 \%$ of our sampled IPD meta-analyses fulfilled the revised criterion in AMSTAR-2 for a comprehensive literature search. Evidence suggests that studies with positive results had a higher probability of getting published in English journals ${ }^{31}$ and that only 5\% of IPD meta-analyses searched non-English databases and 39\% considered non-English publications in their eligibility criteria. IPD meta-analyses therefore might not identify a representative sample of randomised controlled trials. 
Although the impact of a non-representative sample of randomised controlled trials on the effect of IPD meta-analyses is unpredictable and depends on the research topic, publication bias can potentially affect the results and conclusions. ${ }^{8}$ More than two thirds of the sampled IPD meta-analyses in our study did not fulfil the methodological item related to investigation of publication bias. Future researchers should focus on reducing the risk of publication bias through a comprehensive literature search and dealing with its potential impact on the results of IPD meta-analyses.

\section{Risk of bias of included trials}

One recognised advantage of IPD meta-analyses is being able to contact trial investigators when assessing risk of bias to determine the validity of the results. Nonetheless, only $43 \%$ of the sampled IPD metaanalyses used a satisfactory technique to assess the risk of bias in included randomised controlled trials. ${ }^{7}$
The reliability of the evidence derived from an IPD meta-analysis depends on the validity of the included primary studies. ${ }^{32} 33$ Therefore, accounting for risk of bias of included trials during data synthesis, and discussing its potential impact when interpreting the results will help evidence users to judge the confidence of the findings. Furthermore, stratified data synthesis based on risk of bias will provide results from trials exclusively with low risk of bias, which will also facilitate evidence based decision making. However, the sampled IPD meta-analyses in our study performed unsatisfactorily when assessing risk of bias and accounting for it during data synthesis and interpretation of results. Future researchers should deal with these methodological shortcomings when conducting IPD meta-analyses. The updated Cochrane risk of bias tool (RoB2) can be an optimal choice for critical appraisal of included randomised controlled trials. $^{1}$

\begin{tabular}{|c|c|c|c|c|c|c|}
\hline \multirow[b]{2}{*}{ Methodological items } & \multicolumn{2}{|c|}{ Yes } & \multicolumn{2}{|c|}{ Partially } & \multicolumn{2}{|r|}{ No } \\
\hline & $\begin{array}{l}\text { No of IPD } \\
\text { meta-analyses }\end{array}$ & $\%(95 \% \mathrm{Cl})$ & $\begin{array}{l}\text { No of IPD } \\
\text { meta-analyses }\end{array}$ & $\%(95 \% \mathrm{Cl})$ & $\begin{array}{l}\text { No of IPD } \\
\text { meta-analyses }\end{array}$ & $\%(95 \% \mathrm{Cl})$ \\
\hline $\begin{array}{l}\text { I1. Did the research questions and inclusion criteria for } \\
\text { the review include the components of PICO? }\end{array}$ & 274 & 85 (81 to 89 ) & NA & & 49 & 15 (11 to 19$)$ \\
\hline $\begin{array}{l}\text { 12. Did the report of the review contain an explicit } \\
\text { statement that the review methods were established } \\
\text { before conduct of the review and did the report justify } \\
\text { any significant deviations from the protocol?*}\end{array}$ & 99 & $31(26$ to 36$)$ & 109 & $34(29$ to 39$)$ & 115 & $36(30$ to 41$)$ \\
\hline $\begin{array}{l}\text { 13. Did the review authors explain their selection of the } \\
\text { study designs for inclusion in the review? }\end{array}$ & 34 & $10(7$ to 14$)$ & NA & & 289 & $90(86$ to 93$)$ \\
\hline $\begin{array}{l}\text { 14. Did the review authors use a comprehensive } \\
\text { literature search strategy?*}\end{array}$ & 61 & 19 (15 to 23$)$ & 201 & $62(57$ to 68$)$ & 61 & 19 (15 to 23$)$ \\
\hline $\begin{array}{l}\text { 15. Did the review authors perform study } \\
\text { selection in duplicate? }\end{array}$ & 153 & 47 (42 to 53) & NA & & 170 & $53(47$ to 58$)$ \\
\hline $\begin{array}{l}\text { 16. Did the review authors perform data extraction in } \\
\text { duplicate?t }\end{array}$ & 71 & $22(17$ to 26$)$ & NA & & 167 & $52(46$ to 57$)$ \\
\hline $\begin{array}{l}\text { 17. Did the review authors provide a list of excluded } \\
\text { studies and justify the exclusions?* }\end{array}$ & 104 & $32(27$ to 37$)$ & 2 & 1 (0 to 2$)$ & 217 & $67(62$ to 72$)$ \\
\hline $\begin{array}{l}\text { 18. Did the review authors describe the included } \\
\text { studies in adequate detail? }\end{array}$ & 138 & $43(37$ to 48$)$ & 181 & 56 (51 to 62$)$ & 4 & 1 (0 to 2 ) \\
\hline $\begin{array}{l}\text { 19-1. Did the review authors use a satisfactory } \\
\text { technique for assessing RoB in individual studies } \\
\text { that were included in the review?* }\end{array}$ & 139 & 43 (38 to 48 ) & 67 & 21 (16 to 25$)$ & 117 & 36 (31 to 42$)$ \\
\hline $\begin{array}{l}\text { I10. Did the review authors report on the sources of } \\
\text { funding for the studies included in the review? }\end{array}$ & 57 & 18 (14 to 22$)$ & NA & & 266 & 82 (78 to 86$)$ \\
\hline $\begin{array}{l}\text { 112. If meta-analysis was performed, did the review } \\
\text { authors assess the potential impact of RoB in individual } \\
\text { studies on the results of the meta-analysis or other } \\
\text { evidence synthesis? }\end{array}$ & 107 & 33 (28 to 38 ) & NA & & 216 & 67 (62 to 72$)$ \\
\hline $\begin{array}{l}\text { 113. Did the review authors account for RoB in primary } \\
\text { studies when interpreting or discussing the results of } \\
\text { the review?* }\end{array}$ & 128 & 40 (34 to 45$)$ & NA & & 195 & 60 (55 to 66) \\
\hline $\begin{array}{l}\text { 114. Did the review authors provide a satisfactory } \\
\text { explanation for, and discussion of, any heterogeneity } \\
\text { observed in the results of the review? }\end{array}$ & 262 & 81 (77 to 85$)$ & NA & & 61 & 19 (15 to 23 ) \\
\hline $\begin{array}{l}\text { 115. If they performed quantitative synthesis did the } \\
\text { review authors carry out an adequate investigation of } \\
\text { publication bias (small study bias) and discuss its likely } \\
\text { impact on the results of the review? }\end{array}$ & 99 & 31 (26 to 36$)$ & NA & & 224 & 69 (64 to 74) \\
\hline $\begin{array}{l}\text { I16. Did the review authors report any potential } \\
\text { sources of conflict of interest, including any funding } \\
\text { they received for conducting the review? }\end{array}$ & 297 & 92 (89 to 95) & NA & & 26 & 8 (5 to 11$)$ \\
\hline
\end{tabular}

$\mathrm{NA}=$ not applicable; $\mathrm{PICO}=$ population, intervention, comparator, and outcome; $\mathrm{RoB}=$ risk of bias.

${ }^{*}$ Critical item in AMSTAR-2.

tData in this item do not add equal to 323 because 85 IPD meta-analyses are not applicable to the item. 


\begin{tabular}{|c|c|c|c|c|c|c|}
\hline \multirow[b]{2}{*}{ Methodological items } & \multicolumn{2}{|c|}{ Yes } & \multicolumn{2}{|c|}{ Partially } & \multicolumn{2}{|c|}{ No } \\
\hline & $\begin{array}{l}\text { No of IPD } \\
\text { meta-analyses }\end{array}$ & $\%(95 \% \mathrm{Cl})$ & $\begin{array}{l}\text { No of IPD } \\
\text { meta-analyses }\end{array}$ & $\%(95 \% \mathrm{Cl})$ & $\begin{array}{l}\text { No of IPD } \\
\text { meta-analyses }\end{array}$ & $\%(95 \% \mathrm{Cl})$ \\
\hline 19-2. Was the quality of time-to-event-outcome data checked?* & 62 & $19(15$ to 24$)$ & NA & & 123 & $38(33$ to 43$)$ \\
\hline $\begin{array}{l}\text { 111-1. Did researchers stratify or account for clustering of } \\
\text { participants within trials using either a one or two stage } \\
\text { approach to meta-analysis? }\end{array}$ & 315 & 98 (96 to 99) & NA & & 8 & $2(1$ to 4$)$ \\
\hline $\begin{array}{l}\text { I11-2. Was the choice of one or two stage analysis specified in } \\
\text { advance or results for both approaches provided, or both? }\end{array}$ & 143 & $44(39$ to 50$)$ & NA & & 180 & $56(50$ to 61$)$ \\
\hline $\begin{array}{l}\text { I17-1. Were IPD obtained from a large proportion of the } \\
\text { eligible trials? }+\end{array}$ & 166 & $51(46$ to 57$)$ & NA & & 126 & $39(34$ to 44$)$ \\
\hline I17-2. Were the reasons for not obtaining IPD provided?* & $60 \ddagger$ & $48(39$ to 56$)$ & NA & & $66 \ddagger$ & $52(44$ to 61$)$ \\
\hline $\begin{array}{l}\text { I17-3. Were there any strategies taken to account } \\
\text { for unavailable IPD?*}\end{array}$ & $21 \ddagger$ & 17 (10 to 23 ) & $51 \ddagger$ & 40 (32 to 49) & $54 \neq$ & 43 (34 to 52$)$ \\
\hline $\begin{array}{l}\text { I18-1. Were the data checked for missing, invalid, } \\
\text { out of range, or inconsistent items? }\end{array}$ & 180 & $56(50$ to 61$)$ & NA & & 143 & $44(39$ to 50$)$ \\
\hline $\begin{array}{l}\text { I18-2. Did the author check any discrepancies with the trial } \\
\text { report (if available)? }\end{array}$ & 180 & $56(50$ to 61$)$ & NA & & 143 & 44 (39 to 50) \\
\hline I18-3. Were any issues queried and, if possible, resolved?* & 179 & $55(50$ to 61$)$ & NA & & 33 & $10(7$ to 14$)$ \\
\hline $\begin{array}{l}\text { I19-1. Were the methods of assessing whether effects of } \\
\text { interventions vary by participant characteristics appropriate?* }\end{array}$ & 228 & 71 (66 to 76$)$ & NA & & 57 & 18 (14 to 22$)$ \\
\hline $\begin{array}{l}\text { 119-2. Was the choice of participant level characteristics and } \\
\text { methods of assessing participant level interactions specified } \\
\text { in advance?* }\end{array}$ & 101 & $31(26$ to 36$)$ & 25 & $8(5$ to 11$)$ & 159 & 49 (44 to 55$)$ \\
\hline $\begin{array}{l}\text { I20. If there was no evidence of a differential effect by trial or } \\
\text { participant characteristic, was emphasis placed on the overall } \\
\text { results?* }\end{array}$ & 113 & 35 (30 to 40$)$ & NA & & 4 & $1(0$ to 2$)$ \\
\hline 121. Were exploratory analyses highlighted as such?§ & 154 & $48(42$ to 53$)$ & NA & & 16 & 5 (3 to 7$)$ \\
\hline 122. Does any report of the results adhere to the PRISMA-IPD?* & 419 & $32(24$ to 40$)$ & NA & & 869 & $68(60$ to 76$)$ \\
\hline \multicolumn{7}{|c|}{$\begin{array}{l}\text { NA=not applicable; PRISMA-IPD=the Preferred Reporting Items for Systematic Reviews and Meta-analysis for Individual Participants Data extension. } \\
{ }^{*} \text { Data in this item do not add equal to } 323 \text { because some IPD meta-analyses are not applicable to the item. } \\
\text { tData in this item do not add equal to } 323 \text { because } 31 \text { (10\%, 6\% to 13\%) IPD meta-analyses did not report related information of the item. } \\
\text { fDenominator is } 126 \text {. } \\
\text { §Data in this item do not add equal to } 323 \text { because } 153 \text { (47\%, 42\% to 53\%) IPD meta-analyses did not report related information of the item. } \\
\text { IDenominator is } 127 \text {. }\end{array}$} \\
\hline
\end{tabular}

\section{IPD retrieval}

Maximising IPD retrieval is generally considered to have the potential to reduce selection bias, and will likely provide more reliable results. ${ }^{28}$ It is not always easy to obtain IPD from all eligible randomised controlled trials or participants; hence, 90\% IPD retrieval, which was used as the cut-off for a large proportion of IPD retrieval, has been proposed as an acceptable target. ${ }^{34}$ Nonetheless, whether the unavailable IPD introduces bias-which might not only relate to the retrieval proportion but also to other factors, such as whether the unavailable IPD is associated with the direction of the effect estimation and whether sufficient power has been reached with the available IPD. These factors have also been considered according to the criteria used in this study. Although it is not always possible to obtain IPD from $90 \%$ or more of eligible participants or trials, authors can at least provide reasons for it and undertake strategies, such as combining aggregate data with IPD as sensitivity analyses and comparing the differences between trials that provide IPD and those that do not. ${ }^{24}$ Such performance was not, however, common among the sampled IPD metaanalyses that did not obtain IPD from $90 \%$ or more of eligible participants or trials. Future studies should address these methodological flaws.

\section{Strengths and limitations of this review}

This study has several strengths. Firstly, we assessed the methodological quality of IPD meta-analysis by including both general methodological items of systematic reviews and IPD meta-analysis specific methodological items. Secondly, we did not restrict the type of disease or intervention during the sampling process, and as such the sampled IPD meta-analyses covered a wide range of clinical areas and interventions. Thirdly, the performance of each individual methodological item was reported in detail to inform where improvement is required for future studies.

Several limitations are worth mentioning. Firstly, no IPD meta-analysis specific critical appraisal tool exists. In this study, we combined the criteria from AMSTAR-2, Tierney and colleagues' guidance, and other related publications. $^{242526}$ This enabled us to capture the general methodological components of systematic reviews as well as those of IPD meta-analysis. ${ }^{35}$

Secondly, the operational guideline for the criteria we used in our study was developed by adopting rules from AMSTAR- 2 as well as group discussion and consensus. We did not collect any external experts' opinions, nor did we conduct a formal validation process. However, we provided detailed assessment rules for each methodological item to facilitate judgments and strictly followed them. An IPD meta-analysis specific version of the AMSTAR tool is needed in the future.

Thirdly, we only considered the execution of the methodological items, without further inspection of their actual achievements. For example, for the 
risk of bias assessment, we only assessed whether a satisfactory technique was used-such an approach to assess the risk of bias is not equal to assessing the risk of bias appropriately, which was beyond the scope of this study. Likewise, information on statistical methods used for IPD meta-analyses were solely based on the description of the publications. In our study we did not conduct further investigation on whether the statistical methods were applied correctly. Two of the studies indicated that the use of one stage methods were substandard among many IPD metaanalyses. ${ }^{15}{ }^{36}$ Further assessments are warranted on whether clustering was correctly accounted for when the one stage method was used, whether within trial interactions were appropriately separated from across trial interactions to reduce ecological bias when investigating effect modifiers, and whether model assumptions (eg, choice of random or fixed effects) were properly checked. ${ }^{13} 143738$ In addition, no assessment of publication bias is not necessarily equal to the existence of publication bias. The authors of IPD meta-analyses are, however, asked to provide related information to facilitate evidence based decision making. Otherwise, a reassessment of publication bias is needed for evidence users. ${ }^{8}$

Fourthly, owing to limited resources and time, we only sampled and critically appraised IPD metaanalyses that included randomised controlled trials on intervention effects. Conclusions from this study might not apply to IPD meta-analyses including nonrandomised controlled trials or IPD meta-analyses on diagnoses, prognoses, or causes of diseases. Further studies are needed to assess the methodological quality of IPD meta-analyses in these research areas.

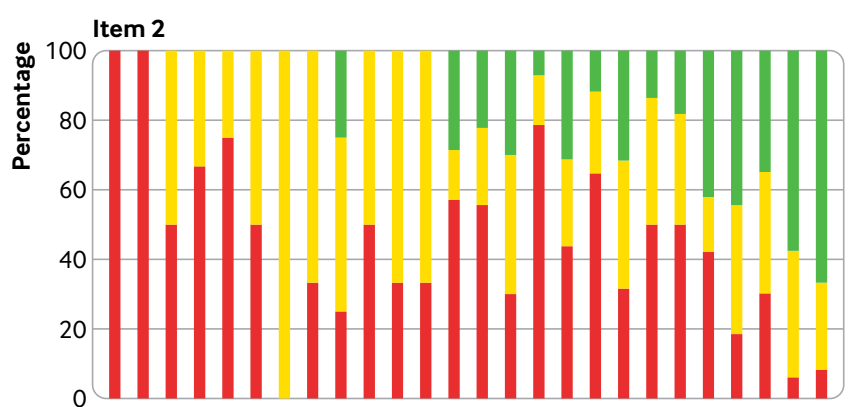

Item 11-2
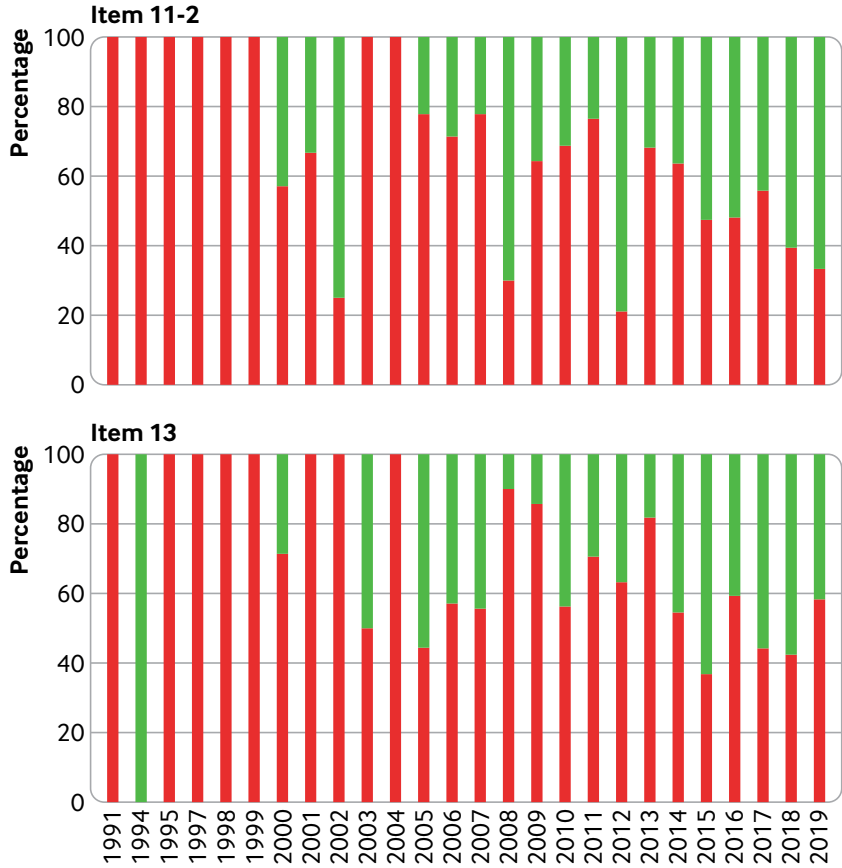

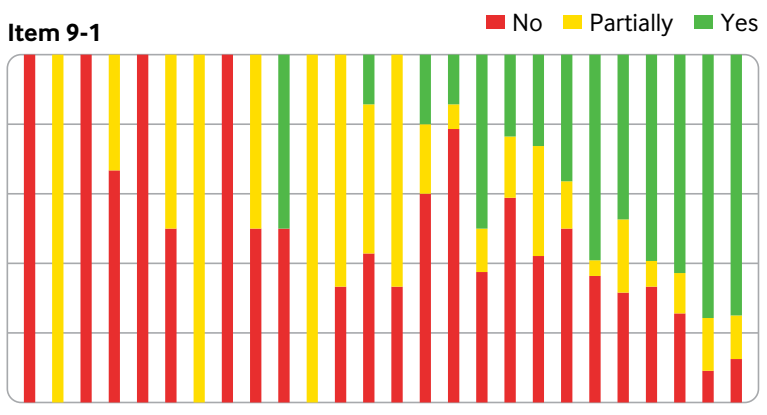

Item 12

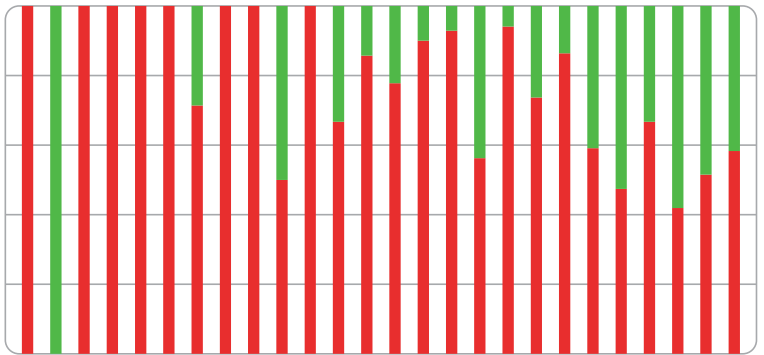

Item 15

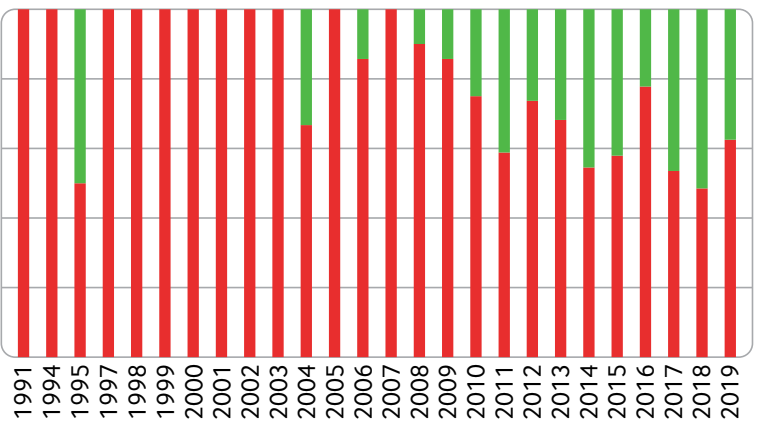

Year

Fig 2 | The methodological quality on six selected items of the 323 sampled individual participant data meta-analyses over time. Item 2: Did the report of the review contain an explicit statement that the review methods were established before conduct of the review and did the report justify any significant deviations from the protocol? Item 9-1: Did the review authors use a satisfactory technique for assessing the risk of bias in individual studies that were included in the review? Item 11-2: Was the choice of one stage or two stage analysis specified in advance or results for both approaches provided? Item 12: If meta-analysis was performed, did the review authors assess the potential impact of risk of bias in individual studies on the results of the meta-analysis or other evidence synthesis? Item 13: Did the review authors account for risk of bias in primary studies when interpreting or discussing the results of the review? Item 15: If they performed quantitative synthesis did the review authors carry out an adequate investigation of publication bias (small study bias) and discuss its likely impact on the results of the review? 
Fifthly, a common drawback of this type of study is that the critical appraisal process relies solely on the reporting of the publications. ${ }^{9}$ Hence, some of the results might be a reflection of the reporting quality instead of methodological quality, especially for the early published IPD meta-analyses. The release of PRISMA-IPD might help improve reporting and facilitate the critical appraisal of future IPD metaanalyses. ${ }^{26}$ Assessing the reporting of the sampled IPD meta-analyses comprehensively is beyond the scope of this study, but it has been covered previously. ${ }^{39}$

Finally, we focused on methodological quality, which is distinguished from the quality of evidence derived from the IPD meta-analyses. The latter is not only affected by the methodological quality of the IPD meta-analyses, but also depends on the features of the primary studies, such as the risk of bias and precision of the effect estimation. ${ }^{20}$

\section{Comparisons with similar studies}

We did not identify any study that comprehensively assessed the methodological quality of IPD metaanalyses. Compared with the methodological quality of aggregate data meta-analyses, the sampled IPD metaanalyses showed better performance in synthesising data with an appropriate method, conducting a comprehensive literature search, and stating conflicts of interests of the review..$^{9-12}$ However, compared with aggregate data meta-analyses, the sampled IPD meta-analyses showed lower compliance in conducting literature selection and data extraction in duplicate, providing adequate details about included randomised controlled trials, assessing the risk of bias with a satisfactory technique and accounting for it during data analyses and interpretation of results, and investigating and discussing publication bias. ${ }^{9-12}$ Discrepancy in the sampling time frame (the past 10 years for aggregated data meta-analyses versus no time restriction for IPD meta-analyses) could have contributed to the observed differences. Reporting might be another reason, as IPD meta-analyses may focus on presenting IPD meta-analyses related details given the word limits of traditional journals; hence, some of the lower compliance might be due to lack of reporting (eg, performance on literature selection and data extraction). The recently developed online appendices policy of many journals and the release of PRISMA-IPD can improve reporting and facilitate the critical appraisal of future IPD meta-analyses. This might contribute to the trends of improvements in the several items observed in this study.

Ahmed and colleagues evaluated publication bias, selection bias, and unavailable data in 31 IPD metaanalyses of randomised controlled trials published between 2007 and 2009. ${ }^{8}$ As with our study, they found similar unsatisfactory performance of comprehensive literature search $(29 \% \quad v \quad 19 \%)$, unsatisfactory consideration of publication bias (32\% v 31\%), and a low proportion ( $52 \% v 51 \%$ ) of meta-analyses collecting IPD from $90 \%$ or more of eligible participants or trials. ${ }^{8}$ We did, however, find trends of improvements in these items, although there is still room for improvement. Compared with the study by Ahmed and colleagues, our study used a much larger sample and comprehensively assessed the methodological quality of included IPD meta-analyses. $^{8}$

Implications for clinical practice and future research Compared with aggregate data meta-analysis, the distinguished features of IPD meta-analysis have made it ideal for systematic review. ${ }^{3}$ It also has a direct impact on healthcare practice and guideline development. ${ }^{5}$ However, results from this study, together with previous related studies, indicated that IPD meta-analysis might not necessarily be free from bias. ${ }^{815} 16$ Therefore clinicians and guideline developers should assess the methodological quality of IPD meta-analyses before making use of the evidence. Researchers should follow the Cochrane Handbook as well as other guidelines for conducting and reporting IPD meta-analysis to ensure the quality of resulting IPD meta-analysis. ${ }^{1} 2 \quad 2426$ An extension specifically for IPD meta-analysis of AMSTAR-2 is needed.

\section{Conclusions}

The methodological quality of IPD meta-analyses is unsatisfactory, either on general items for systematic reviews or on items specific to IPD meta-analyses. Much effort is needed for future IPD meta-analysis in establishing an a priori protocol, prespecifying methods used for data analyses, labelling exploratory analyses, searching literature comprehensively, using an adequate approach to assess the risk of bias of included randomised controlled trials and accounting for risk of bias in data analyses and results interpretation, investigating and discussing publication bias, providing reasons for not obtaining IPD and taking strategies to account for the unavailable IPD, checking data integrity, and clarifying uncertainties. The Cochrane Handbook as well as other methodological guidelines and the PRISMA-IPD statement could be used as reference for future IPD meta-analyses. ${ }^{1} 22426$ It is suggested that the rigour of IPD meta-analyses should be assessed before the results are considered.

\section{We thank Editage for English language editing}

Contributors: IXYW and WT conceived and designed the research questions. IXYW conducted the literature search. HW and YC performed the literature selection. HW, YL, JA, and YC extracted data. HW and YL conducted the critical appraisal. HW analysed data. IXYW and HW interpreted the results. HW wrote the manuscript under the supervision of IW and WT. All authors had full access to all the data in the study and can take responsibility for the integrity of the data and the accuracy of the data analysis. IXYW is the guarantor. The corresponding author attests that all listed authors meet authorship criteria and that no others meeting the criteria have been omitted.

Funding: This research was supported by the High-level Talents Introduction Plan from Central South University (No 502045003) and the National Natural Science Foundation of China (No 81973709). The funders had no role in considering the study design or in the collection, analysis, interpretation of data, writing of the report, or decision to submit the article for publication.

Competing interests: All authors have completed the ICMJE uniform disclosure form at www.icmje.org/coi disclosure.pdf and declare: support from supported by the High-level Talents Introduction Plan from Central South University and the National Natural Science Foundation of China for the submitted work; no financial relationships 
with any organisations that might have an interest in the submitted work in the previous three years; no other relationships or activities that could appear to have influenced the submitted work.

Ethical approval: Not required.

Data sharing: The full dataset is available for review and replication (see supplementary appendix 9).

The lead author (IXYW) affirms that the manuscript is an honest, accurate, and transparent account of the study being reported; that no important aspects of the study have been omitted; and that any discrepancies from the study as planned (and, if relevant, registered) have been explained.

Dissemination to participants and related patient and public communities: The authors plan to disseminate the paper widely to researchers, teachers, clinicians, policy makers, and any other related persons through national and international conferences, webinars, and social media, and by establishing an email discussion group.

Provenance and peer review: Not commissioned; externally peer reviewed.

This is an Open Access article distributed in accordance with the Creative Commons Attribution Non Commercial (CC BY-NC 4.0) license, which permits others to distribute, remix, adapt, build upon this work non-commercially, and license their derivative works on different terms, provided the original work is properly cited and the use is noncommercial. See: http://creativecommons.org/licenses/by-nc/4.0/.

1 Higgins JPT, Thomas J, Chandler J, et al. Cochrane Handbook for Systematic Reviews of Interventions version 6.0 (updated July 2019). Cochrane, 2019. doi:10.1002/9781119536604

2 Tierney JF, Vale C, Riley R, et al. Individual Participant Data (IPD) Metaanalyses of Randomised Controlled Trials: Guidance on Their Use. PLoS Med 2015;12:e1001855. doi:10.1371/journal.pmed.1001855

3 Oxman AD, Clarke MJ, Stewart LA. From science to practice. Meta-analyses using individual patient data are needed. JAMA 1995;274:845-6. doi:10.1001/ jama 1995.03530100085040

4 Nevitt SJ, Marson AG, Davie B, Reynolds S, Williams L, Smith CT. Exploring changes over time and characteristics associated with data retrieval across individual participant data meta-analyses: systematic review. BM/ 2017;357:j1390. doi:10.1136/bmj.j1390

5 Vale CL, Rydzewska LHM, Rovers MM, Emberson JR, Gueyffier F, Stewart LA, Cochrane IPD Meta-analysis Methods Group. Uptake of systematic reviews and meta-analyses based on individual participant data in clinical practice guidelines: descriptive study. BMJ 2015;350:h1088. doi:10.1136/bmj.h1088

6 Tierney JF, Pignon JP, Gueffyier F, et al, Cochrane IPD Meta-analysis Methods Group. How individual participant data meta-analyses have influenced trial design, conduct, and analysis. J Clin Epidemiol 2015;68:1325-35. doi:10.1016/j.jclinepi.2015.05.024

7 Shea BJ, Reeves BC, Wells G, et al. AMSTAR 2: a critical appraisal too for systematic reviews that include randomised or non-randomised studies of healthcare interventions, or both. BMJ 2017;358:j4008. doi:10.1136/bmi.j4008

8 Ahmed I, Sutton AJ, Riley RD. Assessment of publication bias, selection bias, and unavailable data in meta-analyses using individual participant data: a database survey. BMJ 2012;344:d7762. doi:10.1136/bmj.d7762

9 Yan P, Yao L, Li H, et al. The methodological quality of robotic surgical meta-analyses needed to be improved: a cross-sectional study. / Clin Epidemiol 2019;109:20-9. doi:10.1016/j.jclinepi.2018.12.013

10 Tsoi AKN, Ho LTF, Wu IXY, et al. Methodological quality of systematic reviews on treatments for osteoporosis: A cross-sectional study. Bone 2020:139:115541. doi:10.1016/j.bone.2020.115541

11 Wu IXY, Deng Y, Wang H, Chen Y, Wong CHL, Chung VCH. Methodological Quality of Systematic Reviews and Meta-Analysis on Asthma Treatments. A Cross-Sectional Study. Ann Am Thorac Soc 2020;17:949-57. doi:10.1513/AnnalsATS.202003-1870C

12 Wu IXY, Wang H, Zhu L, et al. Methodological quality of systematic reviews on interventions for osteoarthritis: a cross-sectional study. Ther Adv Musculoskelet Dis 2020;12:X20959967.

13 Abo-Zaid G, Guo B, Deeks J), et al. Individual participant data metaanalyses should not ignore clustering. I Clin Epidemiol 2013;66:865873.e4 doi:10.1016/j.jclinepi.2012.12.017

14 Burke DL, Ensor J, Riley RD. Meta-analysis using individual participant data: one-stage and two-stage approaches, and why they may differ Stat Med 2017;36:855-75. doi:10.1002/sim.7141

15 Simmonds MC, Higgins JP, Stewart LA, Tierney JF, Clarke MJ, Thompson SG. Meta-analysis of individual patient data from randomized trials: a review of methods used in practice. Clin Trials 2005;2:209-17. doi:10.1191/1740774505cn087oa

16 Simmonds M, Stewart G, Stewart L. A decade of individual participant data meta-analyses: A review of current practice. Contemp Clin Trials 2015:45(Pt A):76-83.
17 Churuangsuk C, Kherouf M, Combet E, Lean M. Low-carbohydrate diets for overweight and obesity: a systematic review of the systematic reviews. Obes Rev 2018;19:1700-18. doi:10.1111/obr.12744

18 Luo Y-N, Zheng Q-H, Liu Z-B, Zhang F-R, Chen Y, Li Y. Methodological and reporting quality evaluation of systematic reviews on acupuncture in women with polycystic ovarian syndrome: A systematic review. Complement Ther Clin Pract 2018;33:197-203. doi:10.1016/j.ctcp.2018.10.002

19 Gao Y, Cai Y, Yang K, et al. Methodological and reporting quality in non-Cochrane systematic review updates could be improved: a comparative study. J Clin Epidemiol 2020;119:36-46.

20 Balshem H, Helfand M, Schünemann HJ, et al. GRADE guidelines: 3. Rating the quality of evidence. / Clin Epidemiol 2011:64:401-6. doi:10.1016/j.jclinepi.2010.07.015

21 Murad MH, Asi N, Alsawas M, Alahdab F. New evidence pyramid. Evid Based Med 2016;21:125-7. doi:10.1136/ebmed-2016-110401

22 Montori VM, Wilczynski NL, Morgan D, Haynes RB, Hedges Team. Optimal search strategies for retrieving systematic reviews from Medline: analytical survey. BMJ 2005;330:68. doi:10.1136/bmj.38336.804167.47

23 Wilczynski NL, Haynes RB, Hedges Team. EMBASE search strategies achieved high sensitivity and specificity for retrieving methodologically sound systematic reviews. J Clin Epidemiol 2007:60:29-33 doi:10.1016/i.jclinepi.2006.04.001

24 Riley RD, Lambert PC, Abo-Zaid G. Meta-analysis of individual participant data: rationale, conduct, and reporting. BMJ 2010;340:c221. doi:10.1136/bmj.c221

25 Fisher DJ, Copas AJ, Tierney JF, Parmar MKB. A critical review of methods for the assessment of patient-level interactions in individual participant data meta-analysis of randomized trials, and guidance for practitioners. J Clin Epidemiol 2011;64:949-67. doi:10.1016/j. jclinepi.2010.11.016

26 Stewart LA, Clarke M, Rovers M, et al, PRISMA-IPD Development Group. Preferred Reporting Items for Systematic Review and MetaAnalyses of individual participant data: the PRISMA-IPD Statement JAMA 2015;313:1657-65. doi:10.1001/jama.2015.3656

27 Jüni $P$, Witschi A, Bloch R, Egger M. The hazards of scoring the quality of clinical trials for meta-analysis. JAMA 1999;282:1054-60. doi:10.1001/jama.282.11.1054

28 Stewart L, Moher D, Shekelle P. Why prospective registration of systematic reviews makes sense. Syst Rev 2012;1:7. doi:10.1186/2046-4053-1-7

29 Booth A, Clarke M, Dooley G, et al. The nuts and bolts of PROSPERO: an international prospective register of systematic reviews. Syst Rev 2012;1:2. doi:10.1186/2046-4053-1-2

30 Cochrane. Cochrane Library: John Wiley; 2020. https://www. cochranelibrary.com/.

31 Moher D, Pham B, Lawson ML, Klassen TP. The inclusion of reports of randomised trials published in languages other than English in systematic reviews. Health Technol Assess 2003;7:1-90.

32 Debray TP, Moons KG, van Valkenhoef G, et al, GetReal Methods Review Group. Get real in individual participant data (IPD) meta-analysis: a review of the methodology. Res Synth Methods 2015:6:293-309. doi:10.1002/jrsm.1160

33 Savović J, Jones HE, Altman DG, et al. Influence of reported study design characteristics on intervention effect estimates from randomized, controlled trials. Ann Intern Med 2012;157:429-38. doi:10.7326/0003-4819-157-6-201209180-00537

34 Stewart LA, Clarke MJ, Cochrane Working Group. Practical methodology of meta-analyses (overviews) using updated individual patient data. Stat Med 1995:14:2057-79 doi:10.1002/sim.4780141902

35 Stewart LA, Tierney JF. To IPD or not to IPD? Advantages and disadvantages of systematic reviews using individual patient data. Eval Health Prof 2002;25:76-97. doi:10.1177/0163278702025001006

36 Abo-Zaid G, Sauerbrei W, Riley RD. Individual participant data metaanalysis of prognostic factor studies: state of the art?BMC Med Res Methodol 2012;12:56. doi:10.1186/1471-2288-12-56

37 Fisher DJ, Carpenter JR, Morris TP, Freeman SC, Tierney JF. Meta-analytical methods to identify who benefits most from treatments: daft, deluded, or deft approach?BMJ 2017;356:j573. doi:10.1136/bmj.j573

38 Hua H, Burke DL, Crowther MJ, Ensor J, Tudur Smith C, Riley RD. One-stage individual participant data meta-analysis models: estimation of treatment-covariate interactions must avoid ecological bias by separating out within-trial and across-trial information. Stat Med 2017;36:772-89. doi:10.1002/sim.7171

39 Wu IXY, Xiao F, Wang H, et al. Trials number, funding support, and intervention type associated with IPDMA data retrieval: a crosssectional study. J Clin Epidemiol 2021;130:59-68. doi:10.1016/j. jclinepi.2020.10.011

Supplementary information: additional material supplied by authors

Supplementary information: appendix 9 showing full dataset 